\title{
Topical diclofenac in the treatment of osteoarthritis of the knee
}

\author{
This article was published in the following Dove Press journal: \\ Orthopedic Research and Reviews \\ 4 February 20I I \\ Number of times this article has been viewed
}

\author{
Niklas Schuelert \\ Fiona A Russell \\ Jason J McDougall \\ Department of Physiology and \\ Pharmacology, University of Calgary, \\ Calgary, AB, Canada
}

\begin{abstract}
Osteoarthritis (OA) is a common disease that affects millions of people worldwide. As there is no cure for $\mathrm{OA}$, drug treatment to relieve symptoms is the main form of management. Nonsteroidal anti-inflammatory drugs (NSAIDs) such as diclofenac are the most commonly used drugs to treat knee OA pain. Unfortunately, these agents are associated with gastrointestinal and cardiovascular risks, which limit their chronic use. Topical NSAIDs are emerging as a viable alternative for managing OA pain. Because a pharmacologically effective dose can be restricted to the site of pain, there is superfluous systemic absorption, and the risk of related adverse effects is minimized. This article reviews the currently available preclinical and clinical information on topical diclofenac for the treatment of OA pain, including data from recently published randomized controlled trials regarding the efficacy and safety of topical diclofenac. Most studies confirm topical diclofenac to be as effective as oral diclofenac with significantly reduced side effects; however, the efficacy of NSAIDs is far from optimal, and more research needs to be done to investigate the underlying mechanisms of OA in order to improve treatment options, especially for patients with NSAID-resistant OA pain.
\end{abstract}

Keywords: osteoarthritis, topical diclofenac, NSAID, joint pain

\section{Introduction}

Osteoarthritis (OA) is the most common degenerative joint disorder and affects approximately $10 \%$ of the world's population over the age of 60 years. ${ }^{1} \mathrm{OA}$ is generally thought of as "wear and tear" of the joint, affecting middle-aged and older people; however, joint disease can occur at any age, and its onset can be expedited by factors such as joint injury, obesity, and genetics. ${ }^{2}$ Gender also appears to play a role in OA prevalence, with women twice as likely to develop OA than men. ${ }^{3}$ Commonly affected sites include the hip, knee, hand, and spine, with the highest incidence occurring in the knee. ${ }^{1}$ Typical symptoms of OA include pain in and around the joint, morning stiffness, loss of function, immobility, and joint instability. Onset of symptoms is insidious. Pain is generally worse during motion and at night but can sometimes be alleviated by rest. Patients with OA of the knee often present with altered gait and describe a variety of pain qualities such as a sharp/stabbing pain or a dull constant ache. This chronic joint pain experienced by patients is the reason that OA is one of the leading causes of disability worldwide. ${ }^{4}$ The economic burden of this disease is huge, with indirect costs due to work loss and lower productivity, as well as direct costs of medication and hospital care. ${ }^{5,6}$ These expenditures are set to escalate with the aging population, as the incidence of OA rises sharply with age. Recent estimates suggest that, by $2030,25 \%$ of US adults will suffer from arthritis, and a third of those will be of working age. ${ }^{7}$ submit your manuscript | www.dovepress.com

Dovepress

DOI: $10.2147 /$ ORR.S9685
Orthopedic Research and Reviews 201 I:3 1-8

(C) 201 I Schuelert et al, publisher and licensee Dove Medical Press Ltd. This is an Open Access article which permits unrestricted noncommercial use, provided the original work is properly cited. 
The etiology of OA is obscure, and there is no cure for this disease, which is characterized by the breakdown of cartilage and bone in the joint. The loss of cartilage, together with bone remodelling and osteophyte formation, creates joint incongruencies, which lead to altered joint mechanics, thereby further exacerbating the disease. Despite intensive research into tissue engineering, there are, as yet, no reliable means of regenerating mechanically sound articular cartilage, so OA remains a progressive and irreversible degenerative joint disease.

In the absence of any disease-modifying drugs, current OA therapies focus on alleviating the chronic pain, which, in turn, helps to improve joint function and quality of life. Therapy options use both nonpharmacological and pharmacological approaches Nonpharmacological approaches include weight reduction, exercise, patient education, and joint support. One of the major problems with OA is that the pain restricts mobility and the ability to undertake any form of physical activity. Such inactivity can hasten disease progression and exacerbate joint pain. Thus, nonpharmacological techniques are normally combined with analgesics in an attempt to control the pain sufficiently to allow the individual to maintain freedom of movement.

Nonsteroidal anti-inflammatory drugs (NSAIDs), such as diclofenac, are the most common pharmacological agents used to relieve OA pain. A major drawback of using oral forms of NSAIDs, however, is the high incidence of major adverse effects such as gastrointestinal bleeding, gastric ulceration, and renal failure. ${ }^{8}$ In an attempt to reduce the systemic side effects experienced with oral diclofenac, topical formulations of diclofenac were developed for local application. The rationale was that targeted delivery to the site of tissue damage would reduce pain and inflammation while reducing the risk of systemic complications. This review gives a brief introduction to the pathophysiology of OA pain, the role of NSAIDs, their mechanisms of action, and their risk profile. An overview of the current evidence, highlighting the efficacy of topical formulations of diclofenac for treatment of OA of the knee, is given. Many studies have focused on the benefits of topical diclofenac, but in many patients the drug still does not provide sufficient analgesic relief. Therefore, a discussion on the general problems with current OA pain treatment is explored.

\section{Pathophysiology of OA pain}

Joints are complex mechanosensory organs with an elaborate nerve supply. Innocuous mechanical stimuli such as touch, vibration, and pressure are sensed by a population of sensory nerve fibers classed as $A \beta$ fibers. $A \beta$ fibers have large cell bodies ( $>10 \mu \mathrm{m}$ in diameter) and myelinated axons with fast conduction velocities, and activation of these fibers does not normally result in pain detection. ${ }^{9}$ The other population of sensory fibers are the nociceptive nerve fibers, which sense pain and can be divided into two groups: type III (or A $\delta$ ) and type IV (or C). ${ }^{10}$ These fibers have relatively smaller diameter cell bodies with a slower conduction velocity. Nociceptive nerves have a high activation threshold and normally respond only to noxious stimuli. Type III fibers have thinly myelinated axons with unmyelinated "free" nerve endings and, when stimulated, generate "sharp" pain sensations. In contrast, type IV fibers have unmyelinated, slowly conducting axons that transmit dull, "aching" pain information. In the joint, approximately $80 \%$ of afferent nerve fibers are nociceptive, ensuring that the joint is ideally poised to sense potentially harmful movement. Under normal conditions, a painful stimulus, such as an abnormal movement, leads to the activation of specialized receptors on the peripheral terminals of these high-threshold nociceptive primary afferent neurons. The subsequent opening of voltage-gated ion channels triggers an action potential, which conveys the signal from the peripheral nerve terminals to the spinal cord. This signal is then transmitted to dorsal horn neurons and then relayed via projection neurons to higher centers of the brain, resulting in the perception of transient, localized pain. ${ }^{11,12}$ This process warns the individual of the abnormal movement in order to prevent further harm. During OA, changes occur in the pain pathways, leading to prolonged maladaptive pain that often proceeds without any obvious external stimulus. Pronociceptive mediators released into an arthritic joint can trigger excitation and sensitization of peripheral nerves, leading to a heightened pain response. Although our knowledge of the neurobiological processes responsible for the generation of OA pain is still rudimentary, peripheral and central sensitization of neurons is thought to play a major role in the development of pain hypersensitivity observed in OA joints. ${ }^{13,14}$ Erosion of articular cartilage in the OA joint leads to the release of proinflammatory and pronociceptive mediators, which contribute to peripheral nerve sensitization. Because nociceptive sensory fibers innervate most tissues of the joint, pain originates in numerous sites, such as the subchondral bone, periosteum, joint capsule, and synovium.

\section{Nonsteroidal anti-inflammatory drugs: mechanisms of action}

NSAIDs inhibit the cyclo-oxygenase (COX) enzymes, thus preventing the production of prostaglandins. Prostaglandins 
are derived from arachidonic acid, which is released from phospholipids in the cell membrane. The COX enzymes exist in two isoforms: COX-1 and COX-2. COX-1 is constitutively expressed in tissues, whereas COX-2 is typically induced by inflammation. COX-2 is mainly responsible for the breakdown of arachidonic acid to generate prostaglandin $\mathrm{H}_{2}\left(\mathrm{PGH}_{2}\right) . \mathrm{PGH}_{2}$ can then be converted into various other prostaglandins by the action of multiple tissue-specific synthases. ${ }^{15}$ Prostaglandins are released in large amounts under conditions of tissue damage and inflammation, where they can heighten the sensitivity of primary afferent nerve fibers, leading to pain. Prostaglandins can also act on pre- and post-synaptic receptors in the dorsal horn, enhancing membrane excitability and contributing to central sensitization. ${ }^{16}$ These mediators also have various proinflammatory actions; thus, the ability of NSAIDs to prevent prostaglandin production helps to alleviate pain and inflammation.

The first known NSAID, aspirin, functions by acetylating serine residues in the COX enzymes, thus irreversibly inhibiting the enzymes. In contrast, the majority of the newer NSAIDs, including diclofenac, reversibly inhibit COX. Diclofenac is a nonselective COX inhibitor, meaning that it can inhibit both COX-1 and COX-2 isoforms. Originally, it was believed that the analgesic effects of NSAIDs were mediated mainly through inhibition of COX-2. ${ }^{17}$ The concurrent inhibition of COX-1 was thought to bring about the unwanted side effects of NSAIDs, which include gastrointestinal problems due to the inhibition of gastric cytoprotective prostaglandins. Mild gastric problems are fairly common with NSAID use, but serious complications and even death can also occur and are thought to affect over $1 \%$ of arthritic patients taking NSAIDs. ${ }^{8}$ Therefore, there was huge interest in the development of selective COX-2 inhibitors, as it was proposed that these would lack the gastrointestinal side effects. Indeed, several large-scale trials reported that selective COX-2 inhibitors, such as rofecoxib (marketed as Vioxx $^{\circledR}$; Merck \& Co. Inc., Whitehouse Station, NJ), reduced the risk of gastrointestinal events compared with nonselective NSAIDs. ${ }^{18,19}$ However, this was quickly overshadowed by the discovery of the increased risk of serious cardiovascular events with use of these selective inhibitors, which led to the worldwide withdrawal of rofecoxib by Merck Sharp \& Dohme Limited in $2004 .^{20}$ Controversy still remains over other selective COX-2 inhibitors still in clinical use, but, recently, further studies have shown that there appears to be the same risk of cardiovascular problems with both selective and nonselective COX inhibitors (with the possible exception of rofecoxib). ${ }^{21}$
Studies have also challenged the established idea that COX-1 is constitutive and that COX-1 inhibition leads to gastric problems whereas COX-2 is induced during inflammation and is not involved in gastric protection. Wallace et al showed that inhibition of both COX-1 and COX-2 is required for gastric damage, and this may be due to inhibition of COX-1, leading to COX-2 upregulation. ${ }^{22,23}$ Experiments have also shown that COX-2 preferential drugs only exhibit anti-inflammatory effects when given at high enough doses to also inhibit COX-1. ${ }^{24}$ It is now believed that inducible forms of COX-1 exist and that COX-2 is constitutively expressed in some areas such as the kidney. ${ }^{25}$ Therefore, the roles for COX-1 and COX-2 are not as simple and distinct as initially thought, so the idea of using selective COX-2 inhibitors to avoid serious gastrointestinal bleeding is not as attractive as it once was.

\section{The rationale for topical NSAIDs}

With these shortcomings in mind, it was necessary to discover a way to maintain the pain-relieving properties of NSAIDs without the risk of dangerous side effects. This led to the development of topical NSAIDs. The primary rationale for applying a topical agent for pain management in OA is based on the notion that the potentially severe side effects associated with systemic oral agents may be avoided in a topical formulation. Other potential advantages include the fact that analgesia may be applied to superficial joints in a targeted fashion. As mentioned previously, one of the sources of OA pain is the sensitization of nociceptive joint afferents. Moreover, to the extent that a topical agent may be associated with fewer side effects than an oral agent, this allows for more flexible dosing. At present, four products containing diclofenac for topical application have been approved by the US Food and Drug Administration for the treatment of OA. Three products (Pennsaid ${ }^{\circledR}[$ Mallinckrodt Inc., Hazelwood, MO], Solaraze ${ }^{\circledR}[$ PharmaDerm, Melville, NJ], Voltaren ${ }^{\circledR}$ [Novatis Pharma Productions $\mathrm{GmbH}$, Wehr, Germany]) contain diclofenac sodium in different formulations and concentrations. One product (Flector Patch ${ }^{\circledR}$; King Pharmaceuticals Inc., Bristol, TN) contains diclofenac epolamine $1 \%$, and the drug is applied via a patch. Although both topical and oral NSAIDs have the same mechanism of action (inhibition of prostaglandin synthesis), topical NSAIDs exert their effects locally in the treated tissues. ${ }^{26}$ The systemic exposure of topical diclofenac is approximately 17 times lower than that of oral administration, and average peak plasma is 158 times lower than an oral agent (Voltaren Gel [package insert], 
Parsippany, NJ, USA: Novartis Consumer Health Inc; 2007). Plasma drug concentrations after topical NSAIDs are less than $10 \%$ of those following oral dosing. ${ }^{27}$ Topical NSAIDs have been approved for many years in Europe for OA pain. Both the European League Against Rheumatism (EULAR) and the Osteoarthritis Research Society International (OARSI) guidelines recommend the use of topical NSAIDs for pain management in hand and knee OA. ${ }^{28,29}$ Furthermore, the latest guidelines issued in 2008 for OA treatment by the National Institute for Health and Clinical Excellence (NICE) in the UK recommend that topical NSAIDs should be considered ahead of oral NSAIDs. Earlier studies examining the use of topical NSAIDs for OA pain suggested that pain relief might persist for only approximately 2 weeks; ${ }^{30}$ however, numerous subsequent studies have demonstrated a more enduring effect, which is likely a result of different formulations providing greater bioavailability. ${ }^{31-34}$

\section{Therapeutic efficacy of topical NSAIDs}

Several clinical trials and systematic reviews exist on topical diclofenac use for the treatment of OA. Table 1 summarizes the outcome of five recently published (2009-2010) randomized controlled trials regarding efficacy and safety of different formulations of topical diclofenac (diclofenac solution, diclofenac epolamine patch, diclofenac sodium gel). ${ }^{31-34}$ OA of the knee was confirmed in all subjects based on standard radiological criteria for OA. Topical diclofenac was compared with vehicle control and/or placebo in four studies $^{31-33,35}$ and with oral diclofenac in one study. ${ }^{34}$ This latter study also included the combination treatment of topical plus oral diclofenac. Changes in pain score in treated knees were determined using the Western Ontario and McMaster Universities Osteoarthritis Index (WOMAC). Other coprimary endpoints were changes in WOMAC physical function. In all studies, topical diclofenac was significantly more effective than vehicle for improving WOMAC pain and WOMAC physical function in patients with primary OA of the knee. The significant improvements in mean scores were seen in studies lasting 4-12 weeks. One study focused on short-term ( 5 hours) pain relief of a topical diclofenac patch. ${ }^{33}$ No significant differences in efficacy were found between topical diclofenac and oral diclofenac during this acute phase. The formulation used to administer diclofenac differs between the various studies; however, all application methods seemed to reach comparable effectiveness as oral administration.

\section{Safety profile of topical diclofenac}

The majority of clinical trials concluded that topical NSAIDs have a high safety margin (Table 1). The majority of adverse effects seen in clinical trials with topical diclofenac were mild or moderate, with application site reactions being the most common side effect in both the diclofenac and placebo/vehicle groups..$^{31,32,34}$ Dermatitis was often reported in these studies, and this has been attributed to the caustic nature of the vehicles used in each preparation. The dryness effect can be expected with a polar solvent that facilitates drug passage through the skin. The adverse gastrointestinal effects seen with oral diclofenac were significantly less common in patients treated with topical application of the drug. ${ }^{34}$ The systemic adverse effects associated with topical diclofenac treatment were generally infrequent and did not differ from placebo in the vast majority of the trials. A drawback of the clinical studies is the relatively short duration of the trials. Trials that last longer than 12 weeks would provide more information about long-term effects of topical diclofenac use. Also, a more detailed assessment of the specific pain symptoms before and after the treatment might give a clearer picture of which patients are suitable candidates for topical diclofenac treatment. One open-label study has looked at the safety of topical diclofenac solution containing dimethyl sulfoxide over 1 year and observed few adverse side effects and no increased risk of cardiovascular events or cataracts. ${ }^{34}$ However, this study was performed on healthy individuals and not patients with OA, so efficacy of the drug over this long time period was not evaluated. In addition, care must be taken when extrapolating safety data from healthy individuals, as comorbidities are often present in OA patients.

\section{Advantages and disadvantages of topical versus oral diclofenac}

The main advantages of using topical diclofenac versus oral diclofenac for the treatment of OA are the reduced gastrointestinal side effects. The outcome of the studies mentioned in this review suggests that topical administration in recommended doses does not produce the plasma concentrations that are associated with systemic adverse effects. It is important to recognize that use of excessive amounts topically may cause systemic adverse effects. ${ }^{36}$ A recent study showed that plasma levels resulting from topical and oral applications of diclofenac according to clinically recommended prescriptions were comparable; however, after topical application the concentration levels were higher in the muscle and lower in the synovial tissues of OA patients compared with orally treated patients. ${ }^{37}$ From these data we can assume that the 


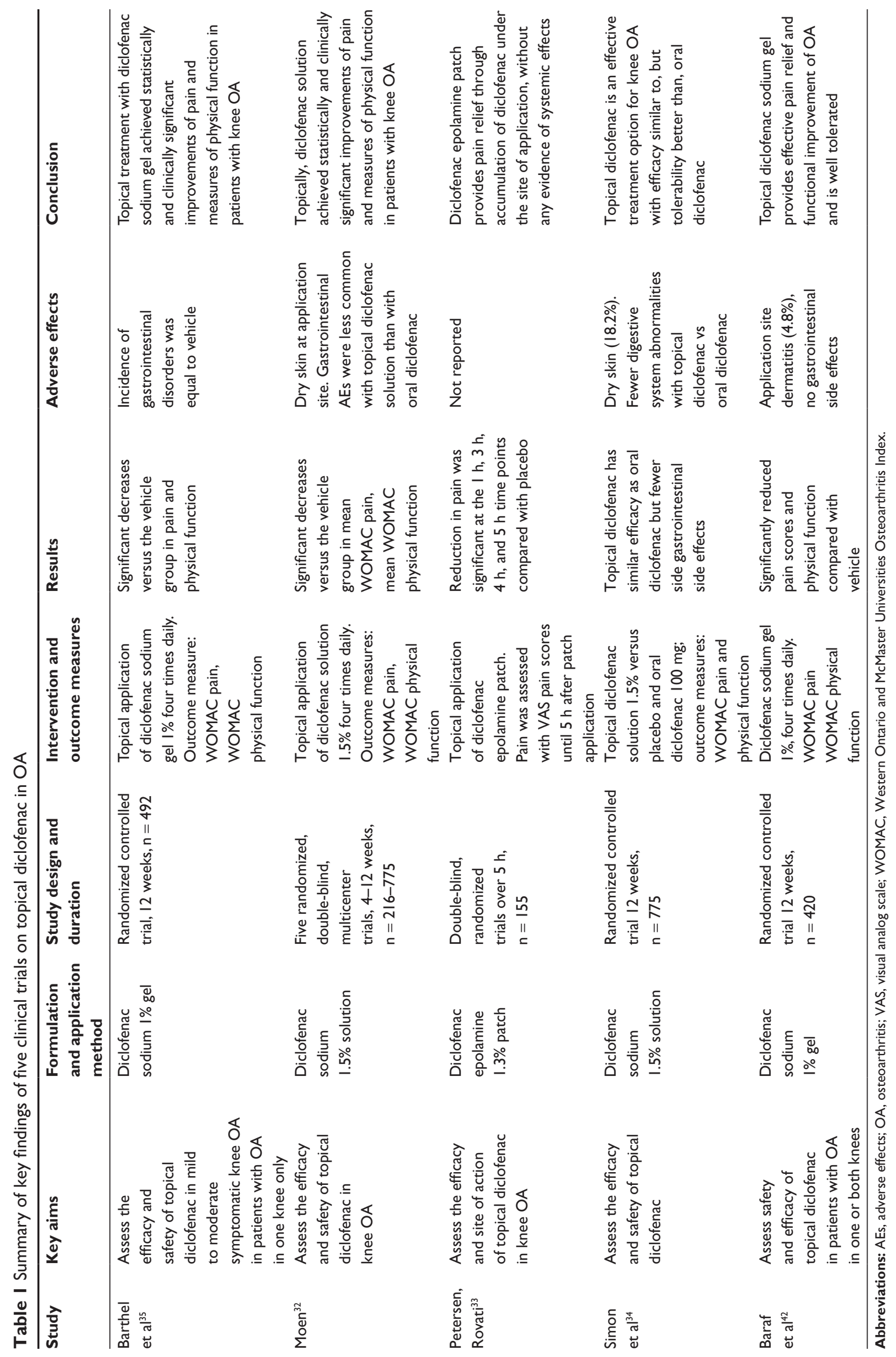


efficacy of topical diclofenac in reducing the symptoms of OA strongly depends on the location and depth of the tissues that are responsible for causing the pain. Unfortunately, most of the published clinical trials evaluated only pharmacodynamic effects of topical diclofenac in OA conditions and did not measure plasma or target tissue concentrations of the NSAID. Therefore, definitive clinical data on the correlation of systemic or tissue diclofenac concentrations and clinical effectiveness are still lacking. Although the cited studies confirm that topical diclofenac is as effective as oral diclofenac for pain relief, the analgesia is still far from sufficient in most patients. Indeed, some patients have no relief at all from either oral or topical NSAIDs. ${ }^{38,39}$

\section{Treatment of osteoarthritis pain remains a challenge}

The management paradigm for pain in OA has changed little over the years. Even though oral and topical NSAIDs are the treatment of choice and can provide some pain relief for OA patients, the level of recovery is not ideal. Some studies show that long-term treatment with NSAIDs does not reduce mean pain levels beyond clinically relevant thresholds. ${ }^{40,41}$ In two of the studies examined in this review, when asked to rate the medication as a treatment for the pain of OA of the knee, approximately $50 \%$ of patients reported topical diclofenac as very good or excellent, whereas $40 \%$ gave the same rating to the vehicle. ${ }^{35,42}$ This was a significant difference, but, in reality, almost the same proportion of patients perceived the control to be as effective a treatment as diclofenac.

A major problem with the management of OA is that the underlying mechanisms that cause the disease and pain are still mostly unknown. Therefore, one potential explanation for suboptimal pain control in OA is a mismatch between the medications used and the underlying pain mechanisms. For example, it is still unknown why OA pain is episodic in some patients, whereas other patients report chronic, persistent joint pain. Another unanswered question is why there is such a substantial disconnect between the degree of joint deterioration and the level of joint pain reported by patients. ${ }^{43-45}$ This is not a subjective issue, as this phenomenon has also been observed in animal models of OA. ${ }^{46}$ Also, the quality of a patient's pain (dull, burning, aching, or stabbing) differs significantly between individuals. ${ }^{47}$ These observations suggest that the etiology of OA is very complex and that OA is not a single disease but a syndrome arising from a group of disorders with similar pathologies.
OA is often referred to as noninflammatory arthritis. This implies that inflammatory processes play a minor role in the pathology of arthritis. However, there are clearly molecular and pathophysiological indices of inflammation in OA that have to be considered. ${ }^{48,49}$ NSAIDs block the COX-2 enzyme, which is the main mediator promoting inflammation, and NSAIDs are still the most prescribed drug for the treatment of OA pain, so we can assume that inflammatory processes are present at least at certain stages of the disease. The different grades of inflammation present at the time of treatment could be the reason why NSAIDs have a pain-relieving effect in some patients and not in others. A major challenge for future research is to understand the basic mechanism of OA pain to find an effective treatment regimen for patients who are unresponsive to oral and topical NSAIDs.

OA pain is considered mainly as nociceptive pain, where cartilage degeneration and pathological changes in subchondral bone lead to inflammation, the release of chemical mediators into the joint, and consequently joint pain. ${ }^{13,14}$ Recent data suggest that people with OA can experience pain due to both nociceptive and neuropathic mechanisms of varying degrees. ${ }^{47,50,51}$ A recent study using animal models of OA showed that neuropathic pain is associated with the disease. ${ }^{52}$ The study infers that cartilage erosion leads to exposure of subchondral bone nerve endings, which under abnormal loading conditions could be damaged, resulting in neuropathic pain. Another possible mechanism of neuropathic pain development is via central sensitization, which can arise from chronic nociceptor stimulation and subsequent modification of central pain-transmitting neurons. ${ }^{53}$ This theory is supported by the fact that some patients still experience joint pain even after complete joint arthroplasty. ${ }^{54}$ Gabapentin, a drug mainly used to treat neuropathic pain conditions, seems to be an effective analgesic in OA pain patients and animal models of joint disease. ${ }^{55,56}$ Whether nerve damage and neuropathic pain are the reason for the lack of pharmacological responsiveness to classic NSAIDs like diclofenac needs further consideration.

In addition to treating the symptoms of OA, a concerted effort has been directed toward slowing down the progression of joint deterioration. Some progress has been made in identifying chemical mediators that are responsible for joint tissue degradation. ${ }^{57,58}$ Chronic inhibition of cathepsin K, for example, has been found to reduce the expression of an OA biomarker as well as attenuating nociception. ${ }^{59}$

To optimize the current treatment of OA, it is crucial that we understand the underlying processes responsible 
for tissue destruction as well as identifying the various neurochemical pathways that are involved in causing pain. It must be recognized that different patients might require different management approaches. A combined therapy to halt disease progression and optimize pain relief individualized for each patient would significantly improve their quality of life.

\section{Conclusion}

There is a wide variety of therapeutic options for the treatment of OA, including pharmacological agents with differing modes of delivery and mechanisms of action, as well as different nonpharmacological approaches. Unfortunately, no single treatment approach is entirely satisfactory, and patients often still suffer from joint pain as well as loss of function. The popularity of oral NSAIDs for the treatment of OA, combined with their propensity to produce risky side effects, has made these particular drugs a cause for concern, especially when used as a long-term monotherapy. The available evidence seems to show that topical diclofenac is as effective as oral diclofenac in relieving joint stiffness and pain. Thus, topical diclofenac shows promise as an effective means of controlling OA pain and appears to be a safer alternative to oral NSAIDs.

\section{Disclosure}

The authors report no conflicts of interest in this work.

\section{References}

1. Woolf AD, Pfleger B. Burden of major musculoskeletal conditions. Bull World Health Organ. 2003;81(9):646-656.

2. Goldring MB, Goldring SR. Osteoarthritis. J Cell Physiol. 2007; 213(3):626-634.

3. Sowers M. Epidemiology of risk factors for osteoarthritis: systemic factors. Curr Opin Rheumatol. 2001;13(5):447-451.

4. Brooks PM. The burden of musculoskeletal disease: a global perspective. Clin Rheumatol. 2006;25(6):778-781.

5. Reginster JY. The prevalence and burden of arthritis. Rheumatology (Oxford). 2002;41(Suppl 1):3-6.

6. Reginster JY, Khaltaev NG. Introduction and WHO perspective on the global burden of musculoskeletal conditions. Rheumatology (Oxford). 2002;41(Suppl 1):1-2.

7. Hootman JM, Helmick CG. Projections of US prevalence of arthritis and associated activity limitations. Arthritis Rheum. 2006;54(1): 226-229.

8. Wolfe MM, Lichtenstein DR, Singh G. Gastrointestinal toxicity of nonsteroidal antiinflammatory drugs. $N$ Engl J Med. 1999; 340(24):1888-1899.

9. Coutaux A, Adam F, Willer JC, Le BD. Hyperalgesia and allodynia: peripheral mechanisms. Joint Bone Spine. 2005;72(5):359-371.

10. Wood JN, Docherty R. Chemical activators of sensory neurons. Annu Rev Physiol. 1997;59:457-482.

11. Kidd BL, Urban LA. Mechanisms of inflammatory pain. Br J Anaesth. 2001;87(1):3-11.

12. Woolf CJ, Costigan M. Transcriptional and posttranslational plasticity and the generation of inflammatory pain. Proc Natl Acad Sci U SA. 1999; 96(14):7723-7730.
13. McDougall JJ. Pain and OA. J Musculoskelet Neuronal Interact. 2006; 6(4):385-386.

14. McDougall JJ. Arthritis and pain. Neurogenic origin of joint pain. Arthritis Res Ther. 2006;8(6):220.

15. Samad TA, Sapirstein A, Woolf CJ. Prostanoids and pain: unraveling mechanisms and revealing therapeutic targets. Trends Mol Med. 2002; 8(8):390-396.

16. Okuse K. Pain signalling pathways: from cytokines to ion channels. Int J Biochem Cell Biol. 2007;39(3):490-496.

17. Griswold DE, Adams JL. Constitutive cyclooxygenase (COX-1) and inducible cyclooxygenase (COX-2): rationale for selective inhibition and progress to date. Med Res Rev. 1996;16(2):181-206.

18. Bombardier C, Laine L, Reicin A, et al. Comparison of upper gastrointestinal toxicity of rofecoxib and naproxen in patients with rheumatoid arthritis. VIGOR Study Group. $N$ Engl J Med. 2000;343(21): $1520-1528,1522$.

19. Silverstein FE, Faich G, Goldstein JL, et al. Gastrointestinal toxicity with celecoxib vs nonsteroidal anti-inflammatory drugs for osteoarthritis and rheumatoid arthritis: the CLASS study: a randomized controlled trial. Celecoxib Long-term Arthritis Safety Study. JAMA. 2000; 284(10):1247-1255.

20. Bresalier RS, Sandler RS, Quan H, et al. Cardiovascular events associated with rofecoxib in a colorectal adenoma chemoprevention trial. N Engl J Med. 2005;352(11):1092-1102.

21. White WB. Cardiovascular effects of the cyclooxygenase inhibitors. Hypertension. 2007;49(3):408-418.

22. Wallace JL, McKnight W, Reuter BK, Vergnolle N. NSAID-induced gastric damage in rats: requirement for inhibition of both cyclooxygenase 1 and 2. Gastroenterology. 2000;119(3):706-714.

23. Tanaka A, Hase S, Miyazawa T, Takeuchi K. Up-regulation of cyclooxygenase- 2 by inhibition of cyclooxygenase-1: a key to nonsteroidal anti-inflammatory drug-induced intestinal damage. $J$ Pharmacol Exp Ther. 2002;300(3):754-761.

24. Wallace JL, Bak A, McKnight W, et al. Cyclooxygenase 1 contributes to inflammatory responses in rats and mice: implications for gastrointestinal toxicity. Gastroenterology. 1998;115(1):101-109.

25. Suleyman H, Demircan B, Karagoz Y. Anti-inflammatory and side effects of cyclooxygenase inhibitors. Pharmacol Rep. 2007;59(3): 247-258.

26. Stanos SP. Topical agents for the management of musculoskeletal pain. J Pain Symptom Manage. 2007;33(3):342-355.

27. Heyneman CA, Lawless-Liday C, Wall GC. Oral versus topical NSAIDs in rheumatic diseases: a comparison. Drugs. 2000;60(3): 555-574.

28. Jordan KM, Arden NK, Doherty M, et al. EULAR recommendations 2003: an evidence based approach to the management of knee osteoarthritis: report of a Task Force of the Standing Committee for International Clinical Studies Including Therapeutic Trials (ESCISIT). Ann Rheum Dis. 2003;62(12):1145-1155.

29. Zhang W, Moskowitz RW, Nuki G, et al. OARSI recommendations for the management of hip and knee osteoarthritis, Part II: OARSI evidence-based, expert consensus guidelines. Osteoarthritis Cartilage. 2008; 16(2):137-162.

30. Lin J, Zhang W, Jones A, Doherty M. Efficacy of topical non-steroidal anti-inflammatory drugs in the treatment of osteoarthritis: meta-analysis of randomised controlled trials. BMJ. 2004;329(7461):324.

31. Baraf HS, Gold MS, Clark MB, Altman RD. Safety and efficacy of topical diclofenac sodium $1 \%$ gel in knee osteoarthritis: a randomized controlled trial. Phys Sportsmed. 2010;38(2):19-28.

32. Moen MD. Topical diclofenac solution. Drugs. 2009;69(18): $2621-2632$.

33. Petersen B, Rovati S. Diclofenac epolamine (Flector) patch: evidence for topical activity. Clin Drug Investig. 2009;29(1):1-9.

34. Simon LS, Grierson LM, Naseer Z, et al. Efficacy and safety of topical diclofenac containing dimethyl sulfoxide (DMSO) compared with those of topical placebo, DMSO vehicle and oral diclofenac for knee osteoarthritis. Pain. 2009;143(3):238-245. 
35. Barthel HR, Haselwood D, Longley S 3rd, et al. Randomized controlled trial of diclofenac sodium gel in knee osteoarthritis. Semin Arthritis Rheum. 2009;39(3):203-212.

36. Zimmerman J, Siguencia J, Tsvang E. Upper gastrointestinal hemorrhage associated with cutaneous application of diclofenac gel. Am J Gastroenterol. 1995;90(11):2032-2034.

37. Miyatake S, Ichiyama H, Kondo E, Yasuda K. Randomized clinical comparisons of diclofenac concentration in the soft tissues and blood plasma between topical and oral applications. Br J Clin Pharmacol. 2009;67(1):125-129.

38. Bjordal JM, Klovning A, Ljunggren AE, Slordal L. Short-term efficacy of pharmacotherapeutic interventions in osteoarthritic knee pain: a meta-analysis of randomised placebo-controlled trials. Eur J Pain. 2007;11(2):125-138.

39. Case JP, Baliunas AJ, Block JA. Lack of efficacy of acetaminophen in treating symptomatic knee osteoarthritis: a randomized, double-blind, placebo-controlled comparison trial with diclofenac sodium. Arch Intern Med. 2003;163(2):169-178.

40. Bjordal JM, Klovning A, Ljunggren AE, Slordal L. Short-term efficacy of pharmacotherapeutic interventions in osteoarthritic knee pain: a meta-analysis of randomised placebo-controlled trials. Eur J Pain. 2007;11(2):125-138.

41. Neame R, Zhang W, Doherty M. A historic issue of the Annals: three papers examine paracetamol in osteoarthritis. Ann Rheum Dis. 2004; 63(8):897-900.

42. Baraf HS, Gold MS, Clark MB, Altman RD. Safety and efficacy of topical diclofenac sodium 1\% gel in knee osteoarthritis: a randomized controlled trial. Phys Sportsmed. 2010;38(2):19-28.

43. Felson DT, Lawrence RC, Dieppe PA, et al. Osteoarthritis: new insights. Part 1: the disease and its risk factors. Ann Intern Med. 2000; 133(8):635-646.

44. Hannan MT, Felson DT, Pincus T. Analysis of the discordance between radiographic changes and knee pain in osteoarthritis of the knee. J Rheumatol. 2000;27(6):1513-1517.

45. Lethbridge-Cejku M, Scott WW Jr, Reichle R, et al. Association of radiographic features of osteoarthritis of the knee with knee pain: data from the Baltimore Longitudinal Study of Aging. Arthritis Care Res. 1995;8(3):182-188.

46. McDougall JJ, Andruski B, Schuelert N, et al. Unravelling the relationship between age, nociception and joint destruction in naturally occurring osteoarthritis of Dunkin Hartley guinea pigs. Pain. 2009; 141(3):222-232.
47. Hochman JR, French MR, Bermingham SL, Hawker GA. The nerve of osteoarthritis pain. Arthritis Care Res (Hoboken). 2010; 62(7):1019-1023.

48. Hedbom E, Hauselmann HJ. Molecular aspects of pathogenesis in osteoarthritis: the role of inflammation. Cell Mol Life Sci. 2002; 59(1):45-53.

49. Pelletier JP, Martel-Pelletier J, Abramson SB. Osteoarthritis, an inflammatory disease: potential implication for the selection of new therapeutic targets. Arthritis Rheum. 2001;44(6):1237-1247.

50. Gwilym SE, Keltner JR, Warnaby CE, et al. Psychophysical and functional imaging evidence supporting the presence of central sensitization in a cohort of osteoarthritis patients. Arthritis Rheum. 2009; 61(9):1226-1234.

51. Im HJ, Kim JS, Li X, et al. Alteration of sensory neurons and spinal response to an experimental osteoarthritis pain model. Arthritis Rheum. 2010;62(10):2995-3005.

52. Ivanavicius SP, Ball AD, Heapy CG, et al. Structural pathology in a rodent model of osteoarthritis is associated with neuropathic pain: increased expression of ATF-3 and pharmacological characterisation. Pain. 2007;128(3):272-282.

53. Woolf CJ. Central sensitization: implications for the diagnosis and treatment of pain. Pain. October 18, 2010. [Epub ahead of print].

54. Toms AD, Mandalia V, Haigh R, Hopwood B. The management of patients with painful total knee replacement. J Bone Joint Surg Br. 2009;91(2):143-150.

55. Hanesch U, McDougall JJ, Pawlak M. Inhibitory effects of gabapentin on rat articular afferent mechanosensitivity. Regulatory Peptides. 2000; 89:63.

56. Buvanendran A, Kroin JS, Della Valle CJ, et al. Perioperative oral pregabalin reduces chronic pain after total knee arthroplasty: a prospective, randomized, controlled trial. Anaesthes Analg. 2010; 110(1):199-207.

57. Cawston TE, Wilson AJ. Understanding the role of tissue degrading enzymes and their inhibitors in development and disease. Best Pract Res Clin Rheumatol. 2006;20(5):983-1002.

58. Rengel Y, Ospelt C, Gay S. Proteinases in the joint: clinical relevance of proteinases in joint destruction. Arthritis Res Ther. 2007; $9(5): 221$.

59. McDougall JJ, Schuelert N, Bowyer J. Cathepsin K inhibition reduces CTXII levels and joint pain in the guinea pig model of spontaneous osteoarthritis. Osteoarthritis Cartilage. 2010;18(10): 1355-1357.
Orthopedic Research and Reviews

\section{Publish your work in this journal}

Orthopedic Research and Reviews is an international, peer-reviewed, open access journal focuses on the patho-physiology of the musculoskeletal system, trauma, surgery and other corrective interventions to restore mobility and function. Advances in new technologies, materials, techniques and pharmacological agents are particularly welcome. The journal welcomes

\section{Dovepress}

original research, clinical studies, reviews \& evaluations, expert opinion and commentary, case reports and extended reports. The manuscript management system is completely online and includes a very quick and fair peer-review system, which is all easy to use. Visit http://www.dovepress. com/testimonials.php to read real quotes from published authors. 